\title{
Containment and Circumscribing Simplices*
}

\author{
E. Lutwak \\ Department of Applied Mathematics and Physics, Polytechnic University, \\ Brooklyn, NY 11201, USA
}

\begin{abstract}
The following containment theorem is presented: If $K$ and $L$ are convex bodies such that every simplex that contains $L$ also contains some translate of $K$, then in fact the body $L$ must contain a translate of the body $K$. One immediate consequence of this theorem is a strengthened version of Weil's mixed-volume characterization of containment.
\end{abstract}

The aim of this note is to prove, and present some simple consequences of, the following:

Theorem. Suppose $K$ and $L$ are convex bodies. If every simplex that contains $L$ contains a translate of $K$, then the body $L$ contains a translate of $K$.

It is helpful to begin by standardizing terminology and notation. It is the author's hope that the reader will find this article to be both self-contained and elementary. Some simple mixed volumes are used, but all the necessary definitions are given, and all the required properties of mixed volumes are established. For general reference, the reader may wish to consult the standard reference by Schneider on the subject of convex bodies and mixed volumes [6].

A convex body is a compact convex subset of Euclidean $n$-space, $\mathbb{R}^{n}$, whose interior is nonempty. A closed half-space in $\mathbb{R}^{n}$ is a set of the form $\left\{x \in \mathbb{R}^{n}: u \cdot x \leq c\right\}$, where $u$ is a unit vector, $c \in \mathbb{R}$, and $u \cdot x$ denotes the usual inner product of $x$ and $u$ in $\mathbb{R}^{n}$. A convex body that is the intersection of $n+1$ closed half-spaces is called a simplex. Thus a simplex has $n+1$ faces and associated with each simplex is the set of $n+1$ outer unit normals to these faces.

A simplex is said to be circumscribed about a convex body if it contains the body and each face of the simplex meets the body. If $\Delta$ is a simplex and $Q$ is a convex body, then $\Delta(Q)$ denotes the simplex circumscribed about $Q$ whose faces have the same set

\footnotetext{
* This research was supported in part by NSF Grant DMS-9507988.
} 
of outer normals as those of $\Delta$. The simplex $\Delta(Q)$ is called the simplex circumscribed about $Q$ corresponding to $\Delta$.

For a convex body $Q$, an $x_{0} \in \mathbb{R}^{n}$, a real $c>0$, and a linear transformation $\varphi \in G L(n)$, the convex body $x_{0}+c \varphi Q$ is defined by

$$
x_{0}+c \varphi Q=\left\{x_{0}+c \varphi x: x \in Q\right\} .
$$

The support function, $h(Q, \cdot): S^{n-1} \rightarrow \mathbb{R}$, of the convex body $Q$ is defined by

$$
h(Q, u)=\max \{u \cdot x: x \in Q\} .
$$

The Hausdorff distance, $\delta(K, L)$, between the convex bodies $K$ and $L$ can be defined by

$$
\delta(K, L)=\max \left\{h(K, u)-h(L, u): u \in S^{n-1}\right\} .
$$

The unit ball centered at the origin is denoted by $B$. The inradius, $r(K)$, of a convex body $K$ is the largest $r>0$ for which there exists an $x \in \mathbb{R}^{n}$ such that $x+r B \subset K$.

A polytope is a convex body that is the intersection of a finite number of closed halfspaces. If $P$ is a polytope with faces that have outer normals $u_{0}, u_{1}, \ldots, u_{s} \in S^{n-1}(s \geq$ $n$ ) and areas $a_{0}, a_{1}, \ldots, a_{s}>0$, then

$$
\sum_{i=0}^{s} a_{i} u_{i}=0
$$

While (1) is easily seen to hold for arbitrary polytopes, it will be used only for the special case where $P$ is a simplex. The mixed volume $V_{1}(P, Q)$, of the polytope $P$ and an arbitrary convex body $Q$, can be defined by

$$
V_{1}(P, Q)=\frac{1}{n} \sum_{i=0}^{s} a_{i} h\left(Q, u_{i}\right) .
$$

If $K$ is an arbitrary convex body, then the mixed volume $V_{1}(K, Q)$ can be defined by approximating $K$ with polytopes. The only mixed volumes that are used in this article are those in which a polytope, in fact a simplex, is the first argument.

A fact regarding mixed volumes that will be needed is that, for a simplex $\Delta$,

$$
V_{1}(\Delta, \Delta)=V(\Delta),
$$

where $V(\Delta)$ denotes the $n$-dimensional volume of $\Delta$. It is easily seen that this is the case for an arbitrary polytope (rather than just a simplex), however, only the case of the simplex will be used.

Proposition. Suppose $\Delta$ is a simplex, $c>0$, and $K$ is a convex body. If a translate of $c \Delta$ circumscribes $K$, then

$$
c=\frac{r(\Delta(K))}{r(\Delta)}=\frac{V_{1}(\Delta, K)}{V(\Delta)} .
$$

In particular,

$$
\Delta(K)=\frac{V_{1}(\Delta, K)}{V(\Delta)} \Delta,
$$

up to translation. 
Proof. Let $u_{0}, u_{1}, \ldots, u_{n} \in S^{n-1}$ denote the outer normals, and let $a_{0}, a_{1}, \ldots, a_{n}>0$ denote the corresponding areas, of the faces of $\Delta$.

Let $H_{i}=\left\{x \in \mathbb{R}^{n}: x \cdot u_{i} \leq h\left(K, u_{i}\right)\right\}$ and let

$$
\Delta^{\prime}=\bigcap_{i=0}^{n} H_{i} .
$$

Clearly, $\Delta^{\prime}$ is a simplex circumscribed about $K$ with the same set of outer unit normals as $\Delta$. Recall that $B$ is the unit ball centered at the origin. Suppose $x, x^{\prime} \in \mathbb{R}^{n}$ and $r, r^{\prime}>0$ are such that $-x+r B \subset \Delta$ is the largest ball contained in $\Delta$ and $-x^{\prime}+r^{\prime} B \subset \Delta^{\prime}$ is the largest ball contained in $\Delta^{\prime}$. Then $B$ is the largest ball contained in the simplices $(1 / r) x+(1 / r) \Delta$ and $\left(1 / r^{\prime}\right) x^{\prime}+\left(1 / r^{\prime}\right) \Delta^{\prime}$. Thus $(1 / r) x+(1 / r) \Delta$ and $\left(1 / r^{\prime}\right) x^{\prime}+\left(1 / r^{\prime}\right) \Delta^{\prime}$ are simplices with the same set of outer unit normals circumscribed about the unit ball centered at the origin. Hence $\Delta^{\prime}=y+c \Delta$ where $y=\left(r^{\prime} / r\right) x-x^{\prime}$ and $c=\left(r^{\prime} / r\right)$.

Now if $y+c \Delta$ is circumscribed about $K$, then

$$
h\left(K, u_{i}\right)=h\left(y+c \Delta, u_{i}\right)=y \cdot u_{i}+c h\left(\Delta, u_{i}\right)
$$

for all $i$. From the definition of the mixed volume $V_{1}$ and (1), we have

$$
\begin{aligned}
n V_{1}(\Delta, K) & =\sum_{i=0}^{n} a_{i} h\left(K, u_{i}\right) \\
& =\sum_{i=0}^{n} a_{i}\left[y \cdot u_{i}+c h\left(\Delta, u_{i}\right)\right] \\
& =y \cdot \sum_{i=0}^{n} a_{i} u_{i}+c \sum_{i=0}^{n} a_{i} h\left(\Delta, u_{i}\right) \\
& =n c V_{1}(\Delta, \Delta) \\
& =n c V(\Delta) .
\end{aligned}
$$

This completes the proof of the proposition.

Suppose $\Delta$ is a simplex, $K$ is a convex body, and $\varphi \in S L(n)$. The proposition shows that a translate of $\left[V_{1}(\Delta, K) / V(\Delta)\right] \Delta$ circumscribes $K$. This implies that a translate of $\left[V_{1}(\Delta, K) / V(\Delta)\right] \varphi \Delta$ circumscribes $\varphi K$. However, by the proposition, a translate of $\left[V_{1}(\varphi \Delta, \varphi K) / V(\varphi \Delta)\right] \varphi \Delta$ circumscribes $\varphi K$. The uniqueness part of the proposition, and the fact that $V(\varphi \Delta)=V(\Delta)$, now yields

$$
V_{1}(\varphi \Delta, \varphi K)=V_{1}(\Delta, K)
$$

The invariance of mixed volumes under simultaneous $S L(n)$ transformations is well known to hold for arbitrary convex bodies. However, (3) is sufficient for the purposes of this article.

Proof of the Theorem. First, note that is it only necessary to prove the theorem for a set of bodies $L$ that is dense in the space of all convex bodies. To see this assume that 
the theorem has been established for all $L \in \mathcal{D}$, where $\mathcal{D}$ is a dense subset of the space of convex bodies. Given an arbitrary convex body $L$, choose $L_{i} \in \mathcal{D}$ such that

$$
L \subset L_{i} \subset L+\frac{1}{i} B=\left\{x+\frac{1}{i} y: x \in L \text { and } y \in B\right\} .
$$

If the hypothesis (of the theorem) holds for $L$, then a fortiori it holds for each $L_{i}$. Hence, for each $i$, there exists an $x_{i} \in \mathbb{R}^{n}$ such that

$$
x_{i}+K \subset L_{i} \subset L+\frac{1}{i} B .
$$

If $x_{o}$ is an accumulation point of the $x_{i}$, then it is easily seen that

$$
x_{o}+K \subset L \text {. }
$$

The dense subset $\mathcal{D}$ will be the set of those polytopes, in $\mathbb{R}^{n}$, no $n$ of whose faces have outer normals that are linearly dependent. Note that if a polytope does not satisfy these conditions, then, by a finite number of small perturbations of its vertices, it can be approximated by polytopes that do meet the conditions.

The theorem is proven by using a standard argument involving Helly's theorem. Recall that Helly's theorem (see, e.g., p. 4 of [6]) states that if $H_{1}, \ldots, H_{s}(s>n)$ are convex sets in $\mathbb{R}^{n}$ with the property that the intersection of any $n+1$ of these sets is nonempty, then the intersection of all $s$ sets is nonempty.

Now suppose $L \in \mathcal{D}$ is a polytope whose faces have outer unit normals $u_{1}, \ldots, u_{s} \in$ $S^{n-1}$. For each $i$, let

$$
H_{i}=\left\{x \in \mathbb{R}^{n}: x \cdot u_{i} \leq h\left(L, u_{i}\right)\right\}
$$

and

$$
K_{i}=\left\{x \in \mathbb{R}^{n}: x+K \subset H_{i}\right\} .
$$

Obviously, the $K_{i}$ are convex and closed.

Suppose $K_{i_{0}}, \ldots, K_{i_{n}}$ are $n+1$ members of the collection $\left\{K_{1}, \ldots, K_{s}\right\}$. Since no $n$ of the vectors $u_{i_{0}}, \ldots, u_{i_{n}}$ are linearly dependent, there are two possibilities: either that $\bigcap_{j=0}^{n} H_{i_{j}}$ contains translates of $r B$ for all $r>0$, or that $\bigcap_{j=0}^{n} H_{i_{j}}$ is a simplex (which obviously contains $L$ ). In the first case there exists an $x_{o} \in \mathbb{R}^{n}$ such that $x_{o}+K \subset$ $\bigcap_{j=0}^{n} H_{i_{j}}$. In the second case $\bigcap_{j=0}^{n} H_{i_{j}}$ is a simplex which contains $L$, so an $x_{o}$ with the property that $x_{o}+K \subset \bigcap_{j=0}^{n} H_{i_{j}}$ is guaranteed by the hypothesis of the theorem. Thus in either case $x_{o} \in \bigcap_{j=0}^{n} K_{i_{j}}$, and Helly's theorem shows that there exists an $x \in \mathbb{R}^{n}$ such that

$$
x \in \bigcap_{i=1}^{s} K_{i} .
$$

Thus $x+K \subset H_{i}$, for $i=1, \ldots, s$, and therefore

$$
x+K \subset \bigcap_{i=1}^{s} H_{i}=L .
$$

This completes the proof. 
An immediate consequence of the theorem is:

Corollary 1. Suppose $K$ and $L$ are convex bodies. If, for each simplex $\Delta$, the simplex $\Delta(L)$ contains a translate of $\Delta(K)$, the $L$ contains a translate of $K$.

An immediate consequence of Corollary 1 is:

Corollary 2. Suppose $K$ and $L$ are convex bodies. If the volume of each circumscribing simplex to $K$ is no greater than that of the corresponding circumscribing simplex to $L$, then some translate of $K$ is contained in $L$.

Corollary 1 can be combined with the proposition to yield immediately:

Corollary 3. Suppose $K$ and $L$ are convex bodies. If

$$
V_{1}(\Delta, K) \leq V_{1}(\Delta, L), \quad \text { for every simplex } \Delta,
$$

then some translate of $K$ is contained in $L$.

The converse of Corollary 3 is an immediate consequence of (1) and the definition of the mixed volume $V_{1}$.

Recently, Scholtes [7] and Zhang [9] strengthened an important (see, e.g., pp. 283284 of [6], p. 151 of [1], and p. 66 of [5]) mixed-volume characterization of containment due to Weil [8]. Scholtes and Zhang proved that Weil's characterization of containment can be strengthened to read: If $K$ and $L$ are convex bodies and if

$$
V_{1}(Q, K) \leq V_{1}(Q, L), \quad \text { for every convex body } Q,
$$

then a translate of $K$ is contained in $L$. Zhang's proof uses Weil's containment theorem and "mixed bodies" (see p. 396 of [6] for a definition).

Weil's proof of his mixed-volume containment theorem is not elementary. In addition to considerable ingenuity, the proof uses information regarding the completion of the space of Aleksandrov-Fenchel-Jessen measures of convex bodies (as well as the fact that every $C^{2}$-function may be represented as the difference of support functions of convex bodies). Clearly, Corollary 3 strengthens Scholtes' and Zhang's result. Clearly also, the proof presented is elementary.

Write $S(Q)$ for the surface area of the convex body $Q$. A question of Fáry and Makai (see p. 22 of [3]) asks: If $K$ and $L$ are convex bodies, and if

$$
S(\varphi K) \leq S(\varphi L), \quad \text { for every } \quad \varphi \in S L(n),
$$

does it follow that $V(K) \leq V(L)$ ?

It was shown in [2] (see also Theorem 4.2.11 of [4]) that the Fáry and Makai question has a negative answer, even if $K$ and $L$ are symmetric about the origin.

Suppose that instead of being told that for each $\varphi \in S L(n)$ the surface area of $\varphi K$ is no greater that that of $\varphi L$, it is given instead that the surface area of a particular simplex circumscribed about $\varphi K$ is no greater than that of the corresponding simplex 
circumscribed about $\varphi L$. Does it follow $V(K) \leq V(L)$ ? Specifically, suppose $\Delta_{o}$ is an arbitrary but fixed simplex. If $K$ and $L$ are convex bodies, and if

$$
S\left(\Delta_{o}(\varphi K)\right) \leq S\left(\Delta_{o}(\varphi L)\right), \quad \text { for all } \varphi \in S L(n)
$$

does it follow that $V(K) \leq V(L)$ ?

Even though the given data appears to be weaker here than in the Fáry and Makai question, it will be shown that this variant of the Fáry and Makai question has a positive answer. No assumptions regarding symmetry need be made. In fact, a much stronger conclusion than $V(K) \leq V(L)$ can be drawn. From the given data, it follows that some translate of $K$ is contained in $L$. Furthermore, the "surface area" in the question can be replaced by "volume" or any other elementary mixed volume (such as "mean width"). Even replacing "surface area" in the question by a functional such as "total edge length," will still lead to the conclusion that a translate of $K$ is contained in $L$. It should be emphasized again that no symmetry assumptions about the bodies involved is needed or will be made.

A translation invariant functional, $\Phi$, defined on the set of simplices, is said to be increasing if, for each pair of positive numbers $c_{1}, c_{2}$,

$$
c_{1}<c_{2} \text { implies } \Phi\left(c_{1} \Delta\right)<\Phi\left(c_{2} \Delta\right),
$$

for each simplex $\Delta$.

Corollary 4. Suppose $\Phi$ is an increasing, translation invariant functional defined on the set of simplices. Suppose that $\Delta_{o}$ is an arbitrary but fixed simplex. If $K$ and $L$ are convex bodies, and if

$$
\Phi\left(\Delta_{o}(\varphi K)\right) \leq \Phi\left(\Delta_{o}(\varphi L)\right), \quad \text { for all } \varphi \in S L(n),
$$

then some translate of $K$ is contained in $L$.

To prove Corollary 4, note that the definition of an increasing functional together with the proposition give

$$
V_{1}\left(\Delta_{o}, \varphi K\right) \leq V_{1}\left(\Delta_{o}, \varphi L\right), \quad \text { for all } \varphi \in S L(n) .
$$

Thus from (3) it follows that

$$
V_{1}\left(\varphi^{-1} \Delta_{o}, K\right) \leq V_{1}\left(\varphi^{-1} \Delta_{o}, L\right), \quad \text { for all } \varphi \in S L(n) .
$$

However, this, and the definition of mixed volumes, shows that $V_{1}(\Delta, K) \leq V_{1}(\Delta, L)$, for every simplex $\Delta$. Corollary 3 now gives the desired result.

\section{Acknowledgments}

The author is grateful to the referees for many helpful comments. Corollary 4, as stated, is due largely to one of the referees. 


\section{References}

1. Yu. D. Burago and V. A. Zalgaller, Geometric Inequalities, Springer-Verlag, Berlin, 1988.

2. G. D. Chakerian and E. Lutwak, On the Petty-Schneider theorem, Contemp. Math 140 (1992), 31-37.

3. H. T. Croft, K. J. Falconer, and R. K. Guy, Unsolved Problems in Geometry, Springer-Verlag, New York, 1991.

4. R. J. Gardner, Geometric Tomography, Encyclopedia of Mathematics, Cambridge University Press, Cambridge, 1995.

5. J. R. Sangwine-Yager, Mixed volumes, in Handbook of Convex Geometry (P. M. Gruber and J. M. Wills, eds.), North-Holland, Amsterdam, 1993, pp. 43-71.

6. R. Schneider, Convex Bodies: the Brunn-Minkowski Theory, Encyclopedia of Mathematics, Cambridge University Press, Cambridge, 1993.

7. S. Scholtes, Minimal pairs of convex bodies in two dimensions, Mathematika 39 (1992), 267-273.

8. W. Weil, Decomposition of convex bodies, Mathematika 21 (1974), 19-25.

9. G. Zhang, Characterizations for zonoids and intersection bodies, preprint.

Received October 31, 1995, and in revised form February 28, 1996. 